\title{
Detection of Hepatocellular Carcinoma in CT Images Using Deep Learning
}

\author{
Shingo Okamoto ${ }^{1}$, Takehiro Yokota ${ }^{2}$, Jae Hoon Lee ${ }^{1}$, Akihiro Takai ${ }^{3}$, Teruhito Kido ${ }^{3}$, Megumi Matsuda ${ }^{3}$ \\ ${ }^{1}$ Graduate School of Science and Engineering, Ehime University \\ Bunkyo-Cho, Matsuyama, Japan \\ okamoto.shingo.mh@ehime-u.ac.jp, lee.jaehoon.mc@ehime-u.ac.jp \\ ${ }^{2}$ Department of Mechanical Engineering, Ehime University \\ Bunkyo-Cho, Matsuyama, Japan \\ ${ }^{3}$ Graduate School of Medicine, Ehime University \\ Shitsukawa, Toon, Japan \\ takai.akihiro.mg@ehime-u.ac.jp, terukido@m.ehime-u.ac.jp, megumi@m.ehime-u.ac.jp
}

\begin{abstract}
The purpose of this paper is to develop a method to detect hepatocellular carcinoma, namely liver cancer in CT (Computerized Tomography) images using the deep learning that is a kind of AI (Artificial Intelligence). Firstly, the learning and recognition programs were developed using Python as a programming language and TensorFlow provided by Google that is a machine learning library. The CT images of 30 clinical subjects were selected from the DICOM format data provided by Graduate School of Medicine of Ehime University. Then 150 sets of CT images were selected where one set consists of two CT images for early and late phases in the cases with hepatocellular carcinoma. In addition, 150 sets of CT images were also selected in the cases without hepatocellular carcinoma. The 450 sets of CT images to each the 150 sets, namely 900 sets in total were created by rotating each original CT image. Consequently, 1,200 sets of CT images (2,400 CT images) in total were used for the learning. Then validity and usefulness of the learning and recognition programs were proved by examining the calculated results. This time, the hepatocellular carcinoma could be detected with relatively high sensitivity of $92.2 \%$ even with a relatively small number of learning data, namely 1,200 sets of CT images.
\end{abstract}

Keywords: Artificial Intelligence, Deep Learning, Computerized Tomography, CT Image, Hepatocellular Carcinoma.

\section{Introduction}

In recent years, performances of medical instruments have improved by the development of science and technology. For example, a multi-slice CT scanner having multiple X-ray detectors has become possible to photograph CT images about 10 times faster than the conventional helical one having a X-ray detector. As the multi-slice CT scanner has also become possible to photograph them by a thinner slice-interval in a wider range, it has become possible to find smaller lesions of two to three millimetres. For these reasons, the diagnosability of lesions using CT images has greatly improved. While, as the CT scanner becomes possible to photograph CT images by a thinner slice-interval in a wider range, radiologists come to have to read so many CT images. Thereby an increase in the burden to radiologists is a problem. Therefore, the development of a CAD (Computer Aided Diagnosis) system is greatly expected.

Then the researches on image recognitions using AI like the deep learning have been also drawn attention in the medical fields. For examples, Junghwan et al. [1] have investigated the influence of learning data size on the learning. In addition, Jianpeng et al. [2] have compared with some models in machine learning. In addition, studies [3], [4] and [5] on applications or their possibilities in the medical field have also been conducted. In addition, studies using medical images are actually conducted. For example, Matthew [6] has conducted a research on hippocampus using MRI data. In addition, Kai et al. [7] have conducted diagnosis of lung cancer using the deep learning. Furthermore, Noorul et al. [8] and Dayong et al. [9] have conducted diagnosis of breast cancer. Furthermore, Yasaka et al. [10] have classified liver masses (masses of cells) using CT images converted to Jpeg format.

In the present research, the TensorFlow provided by Google that is a machine learning library was used. Firstly, the learning program for diagnosis of hepatocellular carcinoma using CT images was developed using the TensolFlow. The 
optimum weight parameters in the learning program were determined examining the calculated results. Next the program to recognize hepatocellular carcinoma was developed using the TensolFlow. Validity and usefulness of the learning and recognition programs were proved by verifying the calculated results.

\section{CNN (Convolution Neural Network)}

Figure 1 shows the schematic chart of CNN (convolutional neural network) used in the present deep learning.

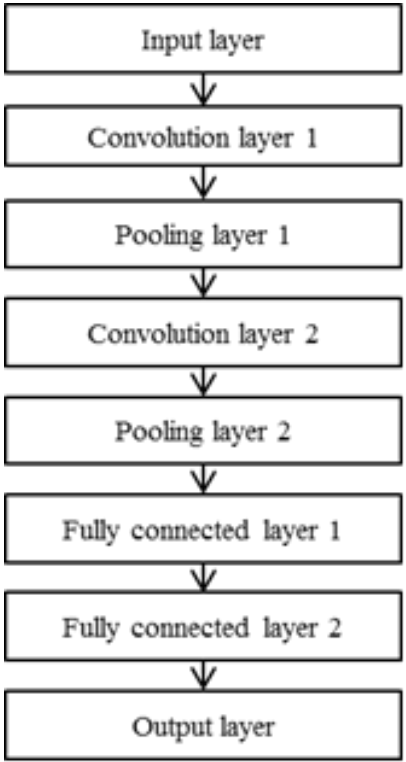

Fig. 1: CNN (Convolutional Neural Network).

\section{Learning Program}

When a contrast medium is injected into a person, the arterial tissue is photographed at early time after 30 to 40 seconds, and the venous one is photographed at late time after around 200 seconds. Radiologists diagnose hepatocellular carcinoma by reading the CT images at both early and late times. Then the CT images at both early and late times were used in the present research. Examples of CT images used in the deep learning are shown in Fig. 2. The CT images of 30 clinical subjects were selected from the DICOM format data provided by Graduate School of Medicine of Ehime University. Then 150 sets of CT images were selected where one set consists of two CT images for early and late phases in the cases with hepatocellular carcinoma. In addition, 150 sets of CT images were also selected in the cases without hepatocellular carcinoma. The 450 sets of CT images to each the 150 sets, namely 900 sets in total were created by rotating each original CT image. Consequently, 1,200 sets of CT images (2,400 CT images) in total were used for the learning. The optimum weight parameters in the learning program were determined examining the calculated results.
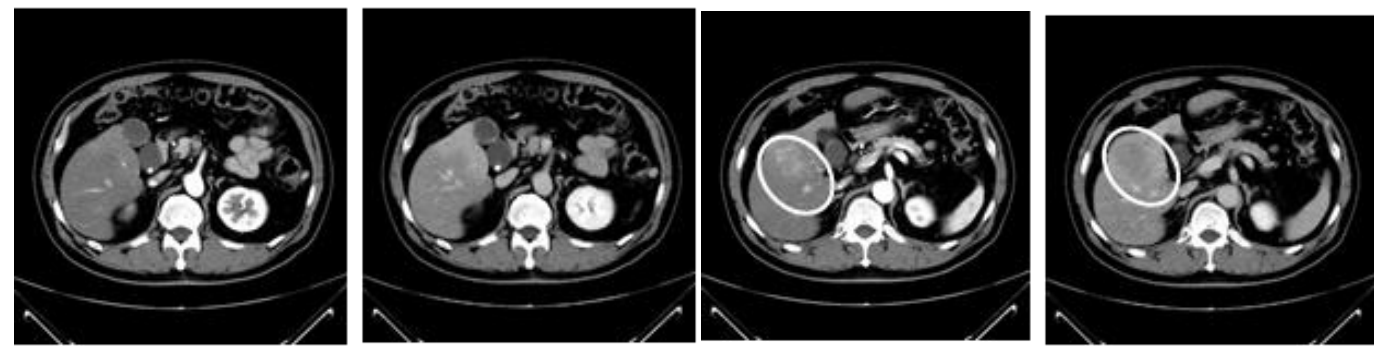

(1) $\mathrm{HCC}(+)$ in early phase
(2) $\mathrm{HCC}(+)$ in late phase White oval marker: HCC (Hepatocellular Carcinoma)

(+): Positive, (-): Negative

Early phase: Hepatic arterial phase, Late phase: Equilibrium phase

Fig. 2: Examples of CT images used in deep learning. 


\section{Recognition Program}

New 232 sets of CT images with hepatocellular carcinoma and new 251 sets of ones without hepatocellular carcinoma that were not used in the learning were used for verification whether the learning was well performed or not. Total of 483 sets of CT images were used for verification. Table 1 shows the examples of results judged by AI (Deep Learning).

\section{Adjustment of Parameters in Deep Learning Program}

The parameters in the deep learning program were adjusted considering the results of ROC (Receiver Operating Characteristic) analysis that is one of methods to evaluate accuracy of the inspection and has been well used in the medical fields. In the present study, the parameters were adjusted by focusing on the sensitivity among three significant values; sensitivity, specificity and AUC (Area Under Curve). In the practical work, the medical statistical software, EZR (Easy R) [11] provide by Jichi Medical University Saitama Medical Center was used for ROC analysis. In the ROC analysis, the cutoff value was set so that both the sensitivity and the specificity can become maximum values. Here the cut-off value means a threshold to identify whether the value obtained by a test is positive or negative.

\section{Learning Results}

The ROC analysis was performed on the probabilities when AI judges that a hepatocellular carcinoma is in each CT image, shown in Table1. The input-image sizes, the filter sizes and the number of learning of CT images were adjusted so that the sensitivity can become larger and the computing time can become shorter.

Firstly, the input-image sizes were adjusted under the conditions where two kinds of filter sizes were fixed by $7 \times 7$ [pixel] and $5 \times 5$ [pixel]. After this, the filter sizes were adjusted under the determined input-image size. We were able to make the sensitivity larger and the computing time shorter when the input-image size and two filter sizes were set to 76×76[pixel], $5 \times 5$ [pixel] and $3 \times 3$ [pixel], respectively. As for these results, the number of learning, the sensitivity and the specificity are shown in Table 2 and Fig.3, respectively.

Table 1: Examples of results judged by AI (Deep Learning).

\begin{tabular}{|c|c|c|c|}
\hline $\begin{array}{c}\text { Image ID } \\
\text { (Early phase) }\end{array}$ & $\begin{array}{c}\text { Image ID } \\
\text { (Late phase) }\end{array}$ & $\begin{array}{c}\text { Diagnosis } \\
\text { by MD }\end{array}$ & $\begin{array}{c}\text { Probability predicted } \\
\text { by AI [\%] }\end{array}$ \\
\hline $01 \_33$ & $01 \_68$ & No $\mathrm{HCC}(-)$ & 39.302 \\
\hline 01_44 & $01 \_79$ & No $\mathrm{HCC}(-)$ & 12.249 \\
\hline $01 \_45$ & 01_80 & No $\mathrm{HCC}(-)$ & 10.200 \\
\hline 0211 & $02 \quad 11$ & No $\mathrm{HCC}(-)$ & 0.633 \\
\hline Jo- & $=0-20$ & rencents & T2.THO \\
\hline $56 \_26$ & $56 \_27$ & $\mathrm{HCC}(+)$ & 70.101 \\
\hline $56 \_27$ & $56 \_28$ & $\mathrm{HCC}(+)$ & 61.187 \\
\hline $56 \_28$ & $56 \_29$ & $\mathrm{HCC}(+)$ & 55.109 \\
\hline $57 \_17$ & $57 \_42$ & $\mathrm{HCC}(+)$ & 97.687 \\
\hline $57 \_21$ & $57 \_46$ & $\mathrm{HCC}(+)$ & 97.855 \\
\hline
\end{tabular}

MD: Medical Doctor, HCC (Hepatocellular Carcinoma)

$(+)$ : Positive, (-): Negative

Early phase: Hepatic arterial phase, Late phase: Equilibrium phase 
Table 2: Parameters in learning.

(Input-image size [pixel]: 76×76, Filter size [pixel]: 5×5, 3×3).

\begin{tabular}{|c|c|c|c|}
\hline No. of learning & Sensitivity & Specificity & Cut-off value \\
\hline 500 & 0.539 & 0.741 & 58.3 \\
\hline 1000 & 0.866 & 0.486 & 39.4 \\
\hline 1500 & 0.871 & 0.510 & 38.3 \\
\hline 2000 & 0.871 & 0.518 & 36.8 \\
\hline 2500 & 0.905 & 0.490 & 30.4 \\
\hline 3000 & 0.888 & 0.498 & 29.0 \\
\hline 3500 & 0.922 & 0.466 & 19.6 \\
\hline 4000 & 0.741 & 0.649 & 54.8 \\
\hline
\end{tabular}

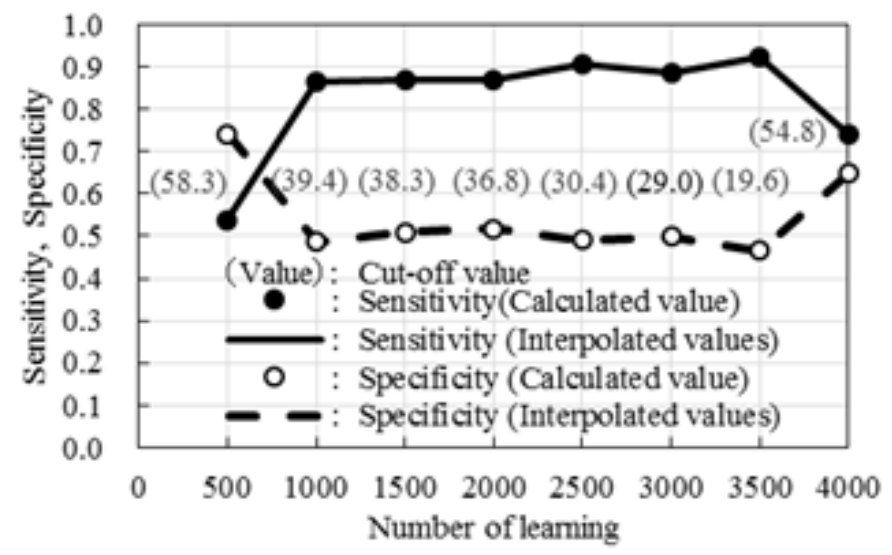

(Input size [pixel]: $76 \times 76$, Filter size [pixel]: $5 \times 5,3 \times 3$ )

Fig. 3: Relation between AUC (Area Under Curve) and Number of learning.

Next, the number of learning was adjusted considering the AUC as well as the sensitivity and the specificity. The relation between the AUC and the number of learning are shown in Table 3 and Fig. 4 when the input-image size and two filter sizes were set to $76 \times 76$ [pixel], $5 \times 5$ [pixel] and 3×3[pixel], respectively. Figures 3 and 4 means that the sensitivity, the specificity and the AUC converge toward the almost constant values from 3,000 times of learning. It was determined that the proper number of learning is 3,500.

Figure 5 shows the ROC curve when the number of learning is 3,500 in the case that the input-image size and two filter sizes were set to $76 \times 76$ [pixel], $5 \times 5$ [pixel] and $3 \times 3$ [pixel], respectively. The cut-off value when the sum of sensitivity and specificity becomes maximum was 19.618 [\%]. Then the sensitivity and the specificity were $92.2 \%$ and $46.4 \%$, respectively when the cut-off value was $19.618 \%$. For reference, the sensitivity and the specificity were $75.92 \%$ and $62.54 \%$, respectively when the cut-off value was $50 \%$. Table 4 shows the classified results in using the cut-off value obtained by ROC analysis. Figure 5 and Table 4 show that hepatocellular carcinoma could be detected with the sensitivity of $92.2 \%$ for 483 sets of CT images. Relatively high sensitivity, $92.2 \%$ was obtained even with a relatively small number of learning data, namely 1,200 sets of CT images. 
Table 3: Number of learning and AUC (Area Under Curve). (Input size [pixel]: 76×76, Filter size [pixel]: $5 \times 5,3 \times 3$ ).

\begin{tabular}{|c|c|}
\hline No. of learning & AUC \\
\hline 500 & 0.696 \\
\hline 1000 & 0.732 \\
\hline 1500 & 0.744 \\
\hline 2000 & 0.749 \\
\hline 2500 & 0.752 \\
\hline 3000 & 0.754 \\
\hline 3500 & 0.756 \\
\hline 4000 & 0.758 \\
\hline
\end{tabular}

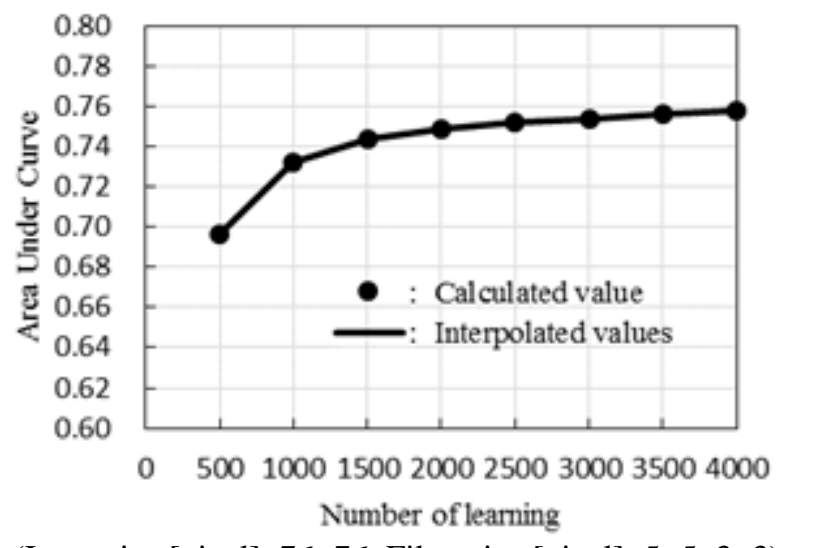

(Input size [pixel]: $76 \times 76$, Filter size [pixel]: $5 \times 5,3 \times 3$ )

Fig. 4: Relation between AUC (Area Under Curve) and Number of learning. 


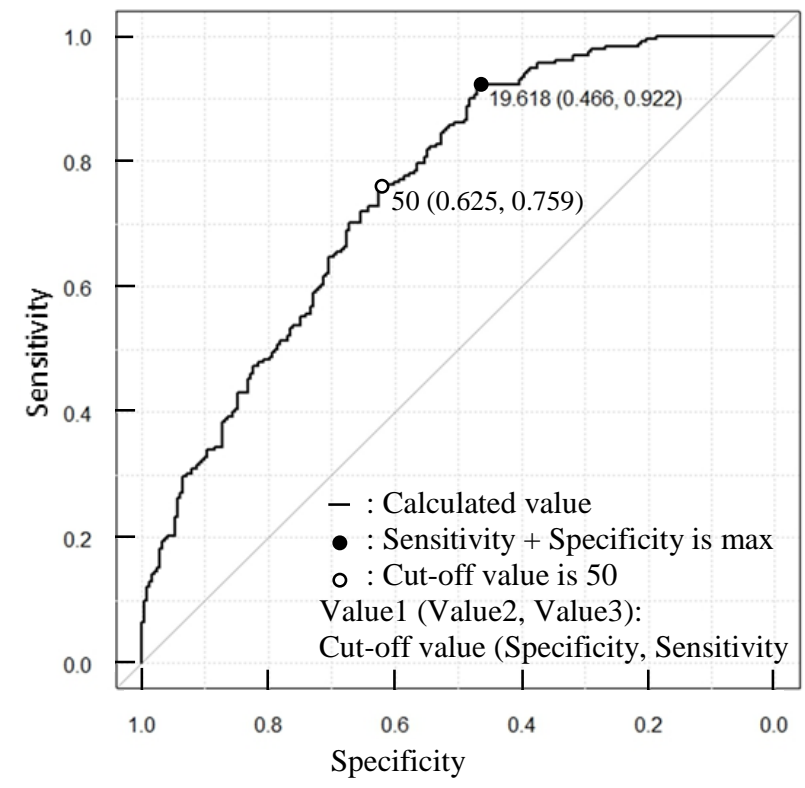

(Input size[pixel]: 76×76, Filter size[pixel]: $5 \times 5,3 \times 3$ )

Fig. 5: ROC (Receiver Operating Characteristic) curve.

Table 4: Classified results (Cut-off Value: 19.618, Sensitivity: 0.922, Specificity: 0.466).

\begin{tabular}{|c|c|c|c|c|}
\hline & \multicolumn{2}{|c|}{ Diagnosis } & \multirow[b]{2}{*}{$\begin{array}{l}\text { Total } \\
\text { [sets] }\end{array}$} \\
\hline & & \multirow{2}{*}{$\begin{array}{c}\begin{array}{c}\mathrm{HCC}(+) \\
{[\text { sets }]}\end{array} \\
214\end{array}$} & \multirow{2}{*}{\begin{tabular}{|c|}
$\begin{array}{c}\text { No } \mathrm{HCC}(-) \\
\text { [sets] }\end{array}$ \\
134 \\
\end{tabular}} & \\
\hline \multirow{2}{*}{ Dignosis } & $\mathrm{HCC}(+)$ [sets] & & & 348 \\
\hline & No $\mathrm{HCC}(-)$ [sets] & 18 & 117 & 135 \\
\hline \multicolumn{2}{|r|}{ Tota1 [sets] } & 232 & 251 & 483 \\
\hline
\end{tabular}

\section{Conclusion}

The research on detection of hepatocellular carcinoma in CT Images using the deep learning was carried out. Firstly, the learning and recognition programs were developed using the Python as a programming language and the TensorFlow provided as a machine learning library. The CT images of 30 clinical subjects were selected from the DICOM format data provided by Graduate School of Medicine of Ehime University. Then 1,200 sets of CT images (2,400 CT images) in total were used for the learning. Validity and usefulness of the learning and recognition programs were proved by examining the calculated results. Finally, the hepatocellular carcinoma could be detected with relatively high sensitivity of $92.2 \%$ even with a relatively small number of learning data, namely 1,200 sets of CT images.

\section{References}

[1] J. Cho, K. Lee, E. Shin, G. Choy, and S. Do, "Medical Image Deep Learning With Hospital Pacs Dataset," Cornell University Library. arxiv.org/abs/1511.06348v1.

[2] J. Zhang, Y. Xia, Q. Wu, Y. Xie, "Classification of Medical Images and Illustrations in the Biomedical Literature Using Synergic Deep Learning,” Cornell University Library. arXiv:1706.09092.

[3] K. B. Wagholikar, V. Sundararajan, A. W. Deshpande, "Modeling Paradigms for Medical Diagnostic Decision Support: A Survey and Future Directions," Springer, US. [Online]. Available: https://doi.org/10.1007/s10916-0119780-4.

[4] G. Litjens, T. Kooi, B. Ehteshami Bejnordi, A. A. Adiyoso Setio, F. Ciompi, M. Ghafoorian, J. A.W.M. van der Laak, B. van Ginneken, C. I. S’anchez, "A Survey on Deep Learning in Medical Image Analysis," Cornell University Library. arXiv:1702.05747. 
[5] A. N. Ramesh, C. Kambhampati, J. R. T. Monson, P. J. Drew, “Artificial intelligence in medicine,” Ann R Coll Surg Engl, vol. 86, pp. 334-338, 2004.

[6] M. Lai, "Deep Learning for Medical Image Segmentation," Cornell University Library. arXiv:1505.02000.

[7] K.-L. Hua, C.-H. Hsu, S. C. Hidayati, W.-H. Cheng, Y.-J. Chen, "Computer-aided classification of lung nodules on computed tomography images via deep learning technique," OncoTargets and Therapy, vol. 8, 2015. https://doi.org/10.2147/OTT.S80733.

[8] N. Wahaba, A. Khana, Y. Soo Lee, "Two-phase deep convolutional neural network for reducing class skewness in histopathological images based breast cancer detection," Computers in Biology and Medicine, vol. 85, pp. 86-97, 2017.

[9] D. Wang, A. Khosla, R. Gargeya, H. Irshad, A. H. Beck, "Deep Learning for Identifying Metastatic Breast Cancer," Cornell University Library. arXiv:1606.05718.

[10] K. Yasaka, H. Akai, O. Abe, S. Kiryu, "Deep Learning with Convolutional Neural Network for Differentiation of Liver Masses at Dynamic Contrastenhanced CT: A Preliminary Study,” Radiology, vol. 285, no. 2, 2017.

[11] Y. Kanda, "Investigation of the freely-available easy-to-use software "EZR" (Easy R) for medical statistics," Bone Marrow Transplant, vol. 48, pp. 452-458, 2013. Advance online publication, 2012. doi: 10.1038/bmt.2012.244. 